\title{
PROCESS AND SYSTEMS The smaller general hospital: Delivering joined up cross-specialty working for the benefit of our patients
}

\author{
Author: Ed Smith ${ }^{\mathrm{A}}$
}

Smaller acute general hospitals, especially those in remote and rural locations, provide vital services to their populations who might otherwise struggle to access safe and effective healthcare. By the nature of their location and, often also reputation, these hospitals are challenging to resource in terms of staffing which makes traditional models of care very difficult to sustain. This article proposes adjustments to the conventional model of acute care that is designed to ensure that patients presenting acutely receive a rapid assessment, according to their medical needs. This is delivered by a multiprofessional team of clinicians delivering care focused on the patient. Hand-offs between clinical teams and duplication of clinical assessment is kept to a minimum. The goal is to deliver care from the most appropriate professional or team as rapidly as possible post presentation, with alternatives to hospital admission being prioritised where appropriate. Early evidence is that this model of care is safe and effective, if delivered within a suitable physical environment for its provision. It is designed to deliver a sustainable model of working for the smaller, remote, rural or challenged healthcare system and is likely to be applicable to such systems elsewhere.

KEYWORDS: Remote, rural, assessment, sustainability, workforce

\section{Introduction}

Making the smaller hospital function well and effectively in the UK is a challenge. If the hospital is rural and/or remote that challenge becomes more acute. There are many reasons for this, but the recurring themes include difficulty in staff recruitment and retention with consequently higher temporary staff costs. Financial challenges provided by funding formulas favouring larger hospitals also compound the difficulties for smaller hospitals. The staffing challenges are particularly taxing for employers. Smaller, more remote hospitals are competing in the same market for staff as larger ones, but have longer travel times from centres of population where the majority of the overall healthcare workforce is located. This is compounded by associated increased travel costs for those staff who do not

Author: ${ }^{\text {A }}$ consultant in emergency medicine, Scarborough Hospital, Scarborough, UK live locally to the hospital. Often such hospitals are in locations of relative deprivation, either rural or coastal, and with a less culturally diverse population. This makes them less attractive areas for potential employees to move to (in terms of community support, schooling and spouse employment). By the time they have completed their training rotation, trainee doctors have often put down roots away from the smaller hospital, having previously chosen to live near a larger centre where they will have spent more time during their specialist training. This makes it much harder to move at this stage (when considering partner employment and children's schooling for instance) which discourages applications to work at the remote site. Unfortunately, the recruitment challenge is self-fulfilling in that trainee medical staff who do choose to work in a smaller hospital often find lower numbers of permanent staff which may lead to a perception of a worse training experience, or increased workload which may discourage them from returning in the future. This clearly exacerbates the problem.

When measured against nationally determined 'standards' of care, the small hospital (and particularly the remote, rural or coastal hospital) frequently struggles. Often this is a function of standards being based on process measures such as staff to patient ratios, rather than outcome measures based on the quality of care delivered. Although there is no evidence that the quality of care delivered by smaller acute providers is worse than that provided in larger institutions, there is a correlation between the rating provided by the Care Quality Commission and the size of the organisation assessed: all those acute hospital trusts in England rated as outstanding in the initial wave of comprehensive inspections of acute trusts were larger than an average trust., ${ }^{1,2}$

The smaller hospital, particularly if it is located in a remote, rural or coastal location, will almost inevitably cost more to staff. There are many components to this; two examples being that the difficulties around recruitment often lead to the employment of a much greater proportion of temporary staff when compared with other centres, and operating essential services (which need to be provided locally because of geography) at a lower scale will incur increased costs. ${ }^{3}$ This budgetary pressure leads to reputational and regulatory pressure which can, in turn, affect recruitment in a 'buyer's' job market as staff move to organisations where they perceive that their role is more sustainable. This doesn't just apply to clinical staff. 


\section{The local challenge}

As a response to the situation described above we have begun to test and develop a new model of care for delivering our acute and emergency services that is designed to enable us to maintain and improve quality and safety in the face of a really challenging staff recruitment and retention environment.

A number of acute services in our hospital are extremely resource constrained in terms of senior medical staff in particular. Emergency department (ED; including urgent treatment centre (UTC)) attendances are around 66,000 per year and medical admission numbers are 45-50 per day. Despite these numbers, we have been in the position of having only 1.4 whole time equivalent emergency medicine consultants, two acute physicians (one post retirement) and three consultant geriatricians. Overall general physician numbers are low and general surgery consultant numbers dipped to two at one point. Paediatrician recruitment and retention has also been extremely challenging.

Often smaller hospitals have more remote locations and as such deliver an essential service to patients presenting who are unable to directly access specialist services (eg renal, vascular and oncology services). In our hospital, these individuals may be holidaymakers or local patients who might typically need to travel 50 miles to routinely access the specialist service. At time of acute need, travel might not be an option and urgent treatment may be required.

Although it continues to evolve, we believe that the core components of the model of care are the key building blocks to delivering sustainable unscheduled care in a resource-challenged environment, and we have begun to assimilate evidence to support the claim.

\section{The principles}

\section{Single point of entry to hospital services}

Having a single point of contact and entry into the service works well for a small volume emergency medical service. This is at odds with conventional wisdom regarding the benefits of streaming patients away from the ED in order to facilitate patient flow and manage pressure on emergency services. Streaming and carving out of services requires all services to be able to function effectively and independently $24 / 7$ and erects barriers between services than can introduce further pressure into an already stressed system. ${ }^{4}$ In a resource-challenged system, it makes sense to consolidate that expertise in one physical location.

Investment in a front-door team that is focused on safety and efficiency pays dividends as it reduces variation in care and makes the implementation of new ways of working significantly easier.

In our system, the single point of entry refers to all adult patients, including those who have been referred in by a healthcare provider (HCP). The exceptions are HCP referred children who arrive directly on the children's assessment unit and women who are in labour who are directed to the delivery suite.

\section{Operate all services 24/7 where-ever possible}

Clearly resource constraints make running services 24 hours a day challenging. However, any interface between the service delivering care in a certain way and changing to deliver care in a different way at any time during a 24-hour period will introduce potential confusion for staff and patients, variation in behaviours and thus unwarranted variation that will potentially impact on quality. Our current example of this is the interface between the 'in hours' hospital mental health liaison team and 'out of hours' mental health crisis team cover which creates a perfectly understandable reticence to see patients towards the end of a shift (and handover to the separately run day team), and a resulting delay in patient care, which could lead to potential patient harm.

We have an on-site 24/7 UTC, staffed by nurse practitioners and general practitioners (GPs) that allows the streaming nurse to refer all patients with minor injury or illness presentations directly to them. The fact that it is run in the same way 24 hours a day makes it easier for the streaming nurses to refer in the same way at all times which minimises variability of response and outcome. This service also functions as the local hub for GP out of hours care.

We believe that a simple system, where the process is unchanged over the 24 -hour period, is a safer system.

\section{Resource the initial assessment process}

The initial clinical contact for walk-in patients is a member of the nursing team who provides a streaming function with only two potential outcomes. The patient is streamed either to the UTC or the emergency stream. If the patient is streamed to the emergency stream (rather than the UTC) by the streaming nurse, or if they have arrived by ambulance, they are seen by the first assessment team. First assessment is akin to rapid assessment and treatment, also referred to as senior doctor triage, early senior assessment or Pitstop. The objective of the process is to put a decision-making clinician in front of the patient as soon as possible in the patient journey. ${ }^{5}$ In many systems, this function is provided by a specialty team or in the ED by a senior doctor, often a consultant. ${ }^{6}$ In our challenged system, we felt that delivery of this function by a consultant was unrealistic and therefore have invested in training and developing a team of advanced clinical practitioners who perform this role in a team approach with the ED senior doctors. The important aspect of the delivery of this role is to understand its function and its limitations - it is simply the first step on the patient journey and does not represent the definitive care of the patient in most cases (except when the patient can be discharged directly from there).

'First assessment' in our service has two main distinct functions: safety and efficiency.

\section{Safety}

This is the primary function of first assessment. Because all patients who are deemed unsuitable for the UTC are streamed into this service, it is the responsibility of the first assessment team to ascertain whether immediate intervention is required (eg airway compromise). This assessment can happen virtually at times of pressure on the system - not all patients have to physically visit the first assessment area (eg if direct access to a side room is required for infection control purposes). However, the team needs to be aware of them and their presentation and ensure that the patient is safe to go on the next stage of their journey.

Having a single assessment process via a single point of entry allows the rapid introduction of new ways of working and an effective focus on the potentially sick patient. The standard for review is within 30 minutes of arrival (our streaming standard is 15 minutes but patients for first assessment will already have been seen either in streaming or by an ambulance crew, hence the slightly longer time standard).

\section{Efficiency}

Once the lack of need for immediate intervention has been established, then the focus of the assessing clinician is on what 
needs to be done now for this patient in order to facilitate a decision to be made about the next steps in care. Typically, this involves requesting simple relevant investigations, eg bloods and plain chest $\mathrm{X}$-ray if there is a suspicion of community acquired pneumonia or computed tomography (CT) of the head if a patient is anticoagulated and has sustained a head injury. Treatment is usually deferred until the next stage in the process as there is a time pressure to move patients through to ensure that the next patient can be processed through first assessment.

The practitioner working in first assessment is then able to ensure that the patient is moved through the system to the most appropriate location and practitioner. This is determined by a set of established principles and agreements drawn up between the specialties as to who looks after which presentations, and is designed to remove the subjectivity and debate from the process. Internal audit work suggests that this decision making is correct in 90-95\% of cases.

The exception to the patient rapidly moving on in this way occurs if there is a critical need for urgent treatment and there is no other location in which to deliver it (eg 1-hour antibiotics in sepsis).

The options for the patient moving on through the system are:

> specialist assessment unit or same day emergency care (SDEC; eg emergency assessment unit or children's assessment)

> discharge directly from first assessment with/without community follow-up.

This ability to direct the patient in the most efficient way is critical to development and delivery of the model and is designed to maximise the opportunity for discharge; consistently $47-49 \%$ of HCP referred patients are discharged home or managed through SDEC services without the need for a formal admission.

Think of the question you are trying to answer and ensure that it is answered by the most appropriate clinician

All patients are given a specialty attribution from first assessment. This is based on a set of detailed agreements between each specialty and the ED as to who 'owns' which patient. These agreements also document the decision as to who is the senior decision maker that is empowered to make a clinician decision about each category of patient. Examples of this include:

$>$ an adult patient who either self-presents or is referred into the hospital with an acute exacerbation of asthma is given a specialty disposition of general medicine; they improve after initial treatment and they are reviewed, and a decision about admission or discharge can be made safely by an specialty trainee $4+(\mathrm{ST} 4+)$ in emergency medicine or general/acute medicine

> a male teenager who either self presents or is referred with right sided lower abdominal pain is given a specialty disposition of general surgery; their pain settles, blood test results are unremarkable and they can be discharged by an ST $4+$ in emergency medicine or general surgery.

An example of such a specialty agreement is given in Fig 1. Repeated audits of the effectiveness and accuracy of the decision making and clinical disposition in first assessment suggest that the assessment is correct in around $90 \%$ of cases (Fig 2).
The hospital patient tracking system enables us to identify the location and specialty disposition of all patients at any one time. Implementation of the system that has been described was demonstrated to deliver an improvement in time to assessment (Fig 3a) and decision making within the service (measured at 2 hours after patient arrival: Fig 3b). It has been challenging to sustain that improvement because exit block and crowding in the ED has impaired the ability of first assessment to function effectively as patients can no longer be consistently moved out of the service to the next stage of their diagnostic and therapeutic journey.

\section{Same day emergency care and home first for frailty}

A relatively high proportion of short stay admission patients can be managed through non-admitted pathways. We believe that the most effective and efficient way of managing these patients is by early clinician review in first assessment, frontloading investigations that will contribute to answering the question asked (eg D-dimer for suspected deep-vein thrombosis given appropriate Wells score pre-test probability) and then using an $80: 20$ rule to decide where the patient should be streamed to for ongoing assessment and decision making, ie if, in the opinion of the assessing clinician, the patient is $80 \%$ likely not to require an admission then they are suitable for SDEC/ambulatory emergency care. Although we don't currently deliver SDEC 24/7 we believe that working towards that goal is appropriate in terms of consistency and reliability of delivery of care.

The practical challenge with many acute patients is that there is great variety in how much time is required in the service to the point at which the patient can be safely or efficiently managed in a nonhospital setting. The difficulty in establishing uniformity of standards around this is the variability in availability of support services such as radiology and therapy services across systems. In one system, with $8 \mathrm{am}-8 \mathrm{pm}$ therapy support for example, it may be possible to ensure safe supported discharge of a frail elderly patient who arrived at $4 \mathrm{pm}$ and does not require hospital admission for ongoing treatment. If they are fit to be assessed at $10 \mathrm{pm}$, it is possible that they cannot be safely discharged at that time, but they can be at 9am the following day. The length of stay in terms of hours in a SDEC service is a product of the nature of the presentation, the hours of support services available and the staffing resource available to manage that patient in a non-admitted setting.

Our service is currently able to provide a therapist assessment of our patients for 12 hours per day, 7 days per week. This is critical in delivering a 'home first' approach to managing our frail elderly patients and ensures that those patients that are admitted are managed in that way because there is genuinely no alternative medically or socially at the time of their presentation.

\section{Single assessment and clerking}

One of the driving principles of the described model of care is to remove duplication of patient assessment and documentation. There are many arguments for this including improving efficiency of the process, reducing the time to decision making about patient care and improving the patient experience by avoiding repetition of questioning. ${ }^{4}$ To this end we have introduced a process of single clerking on the inpatient admission pro forma for all those patients who are deemed at first assessment to have a strong likelihood of needing admission. 
Fig 1. Example of a specialty agreement care pathway. Acute medical model care pathway for trauma and orthopaedic presentations. $\mathrm{ACP}=$ advanced clinical practitioner; $\mathrm{CT}=$ computed tomography; $\mathrm{CPD}=$ core patient database (hospital IT system); CXR = chest $\mathrm{X}$-ray; $\mathrm{ED}=$ emergency department; $\mathrm{G} \& \mathrm{~S}=$ group and save; $\mathrm{MRI}=$ magnetic resonance imaging; spec doc $=$ specialist doctor; $\mathrm{ST} 3+=$ specialty trainee $3+$; $T \& O=$ trauma and orthopaedic; UCC = urgent care centre.

\begin{tabular}{|c|c|}
\hline $\begin{array}{l}\text { Definition of T\&O presentations: } \\
\text { - cauda equina syndrome / suspected (non-malignant) } \\
\text { - child requiring manipulation of a fracture } \\
\text { - compartment syndrome / suspected } \\
\text { - dislocation of hip or prosthesis } \\
\text { - dislocation of any other large joint (aim for } \\
\text { immediate/early reduction where possible) } \\
\text { - dislocation of small joint that cannot be reduced in } \\
\text { UCC } \\
\text { - extensor tendon injuries to the hand (open) } \\
\text { - foreign body in a limb requiring surgery } \\
\text { - fractured neck of femur } \\
\text { - long bone fracture / likely including tibial plateau } \\
\text { - mecture } \\
\text { temporary inability to mobilise } \\
\text { - occult fractured neck of femur - clinical suspicion } \\
\text { of hip fracture } \\
\text { - discharge prior to diagnosis not appropriate } \\
\text { (eg non-weight bearing) } \\
\text { - open fracture and/or dislocation } \\
\text { - septic arthritis / suspected } \\
\text { mignificant soft tissue injury eg disrupted extensor } \\
\text { mechanism lower limb } \\
\text { - vertebral fracture(s) at any level if further } \\
\text { investigation or inpatient treatment required } \\
\text { eg CT/MRI. }\end{array}$ & $\begin{array}{l}\text { Exclusion criteria to direct T\&O } \\
\text { assessment: } \\
\text { - alternative (eg medical) cause for } \\
\text { presentation is primary concern } \\
\text { (determined by assessment team) ie } \\
\text { frailty or medical assessment precedes } \\
\text { surgical assessment } \\
\text { - fracture that is incidental to } \\
\text { presentation and not the reason for } \\
\text { admission (eg humeral fracture, unable } \\
\text { to cope at home) } \\
\text { - patient requires resuscitation room } \\
\text { based on acuity of presentation } \\
\text { - major trauma requires trauma } \\
\text { team management (includes T\&O). }\end{array}$ \\
\hline \multicolumn{2}{|c|}{$\begin{array}{l}\text { Key investigations: } \\
\text { - determined by presentation (see supporting pathways) } \\
\text { - typically: bloods = ED general and G\&S if likely to require theatre (coagulation test if on } \\
\text { anticoagulants) } \\
\text { - inflammatory markers if suspected septic joint } \\
\text { - radiography of affected area (CXR if theatre likely and anaesthetic requirement). }\end{array}$} \\
\hline \multicolumn{2}{|c|}{$\begin{array}{l}\text { Referral process / discharge arrangements and lines of clinical responsibility: } \\
\text { - all patients once referred are considered to be under the care of the T\&O team working at the time } \\
\text { the referral is made } \\
\text { the discharging clinician takes responsibility for the decision to discharge the patient, working within } \\
\text { their normal protocols and within the usual limits of their expertise ie patient may be discharged by } \\
\text { ST3+ or equivalent in surgery or emergency medicine - the responsibility sits with the consultant } \\
\text { overseeing the clinical work of that practitioner. }\end{array}$} \\
\hline
\end{tabular}

This has been relatively challenging to implement due to resistance from both sides of the emergency medicine / general medicine interface. Concern from the emergency medicine side has focused on the additional time required to do a 'proper' clerking over and above that which the team was used to. This has been exacerbated by the disproportionately high number of emergency medicine middle grade locums involved in the delivery of the service who tend to deliver a relatively focused and brief documentation style. From the general medicine perspective the issue has been around the quality standard of the work produced, which links to the issue of who has seen the patient. This is managed effectively by ensuring that the clerking team understand where they are in the process and that standard of documentation that is expected of them. Quality improvement work is ongoing to ensure a continued focus on allaying anxieties on both sides and embedding a single team focused on accurate assessment of the needs of the patient.

\section{Training opportunities and experience}

One anxiety about the model of care proposed relates to the opportunity for medical (and indeed advanced clinical practitioner and physician associate) training at all levels, because of the aim to compress the medical assessment into fewer steps and deliver it under the auspices of a single clinical team. We would argue that although there is a single team, it is multiprofessional and as such has ample opportunity to deliver training experiences across the specialties. It supports the move towards a greater focus on generalist training that is described in the new internal medicine training curriculum for general (internal) medicine trainees.

As colleagues work to their skill set, rather than their badge, there is a greater opportunity for trainees in all specialties to learn from experts in others. It is also the case that this system drives an imperative for earlier senior review that should therefore provide immediate feedback to the clerking doctor in today's shift-based world that will enhance the training experience. 


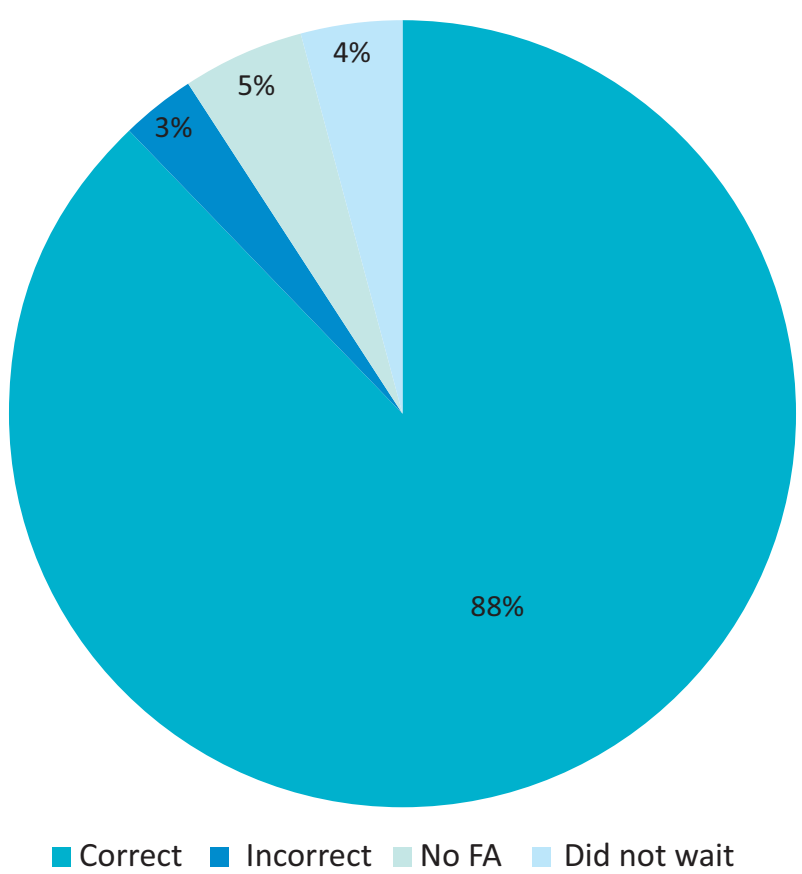

Fig 2. First assessment decision making. Audit data $(n=185)$ from weekend day of all emergency department attends. FA = first assessment.

\section{Culture, behaviour and leadership}

The model of care described above can only be implemented within a supportive cultural, behavioural and leadership framework, and we have learnt the importance of developing that in parallel with the clinical model. There must be trust between teams to accept the patient on face value in terms of what is reported to clinicians further along the patient journey about likely pathology as well as acuity. Audits of the first assessment process suggest that clinicians working within it comply with the 'rules' (ie get the specialty disposition correct) in about $90 \%$ of cases (Fig 2). Analysis of serious incidents suggests that almost always the clinician closest to the start of the process is able to identify that the patients that are seriously unwell. Difficulties tend to arise subsequently when those involved further along the patient journey do not respond in the appropriate way to the message that is being delivered from the point of initial assessment. This has been particularly challenging with individuals who have rotated in from other systems that work in a different way, or who are ingrained in a way of working that is delivered from a 'silo' perspective. The majority of the senior and permanent hospital staff have come with us on the journey and are committed to patient-focused delivery of care that we are trying to embed.

\section{Estates}

This model is predicated on co-location wherever possible of all acute services. The argument for that follows.

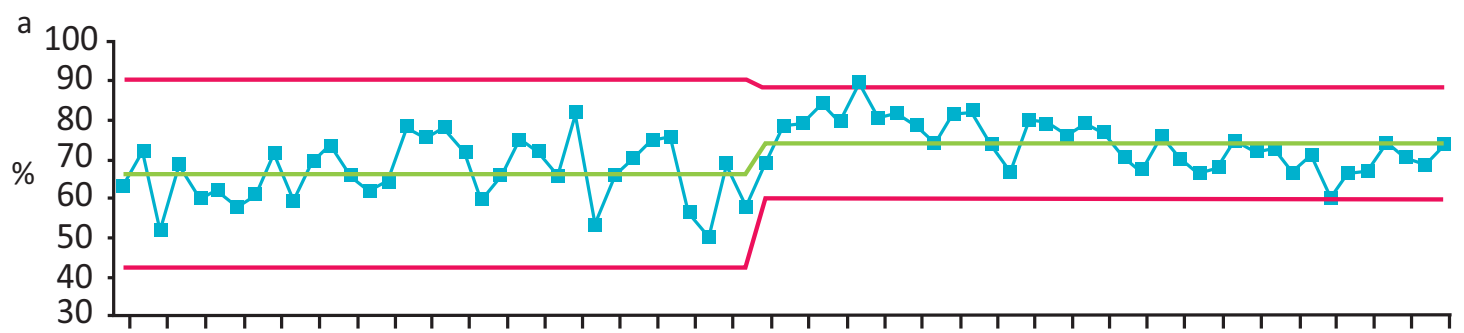

o 홍 ळ ठં

Date

$\rightarrow-$ Proportion seen within 30 mins — Average —UCL and LCL

b

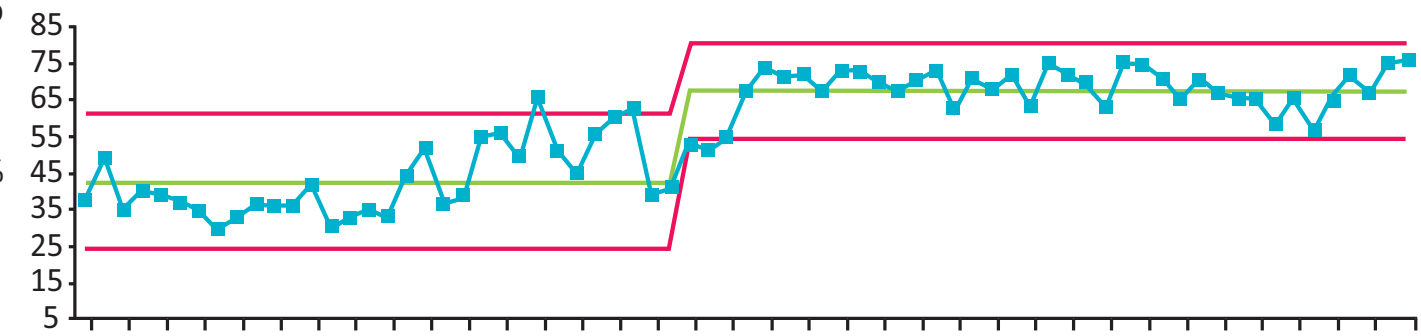

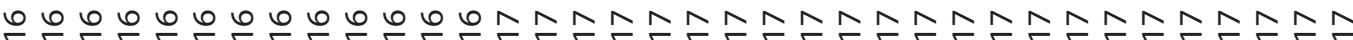

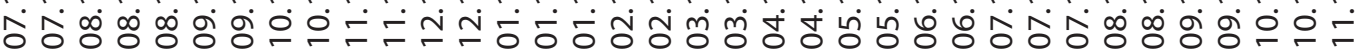

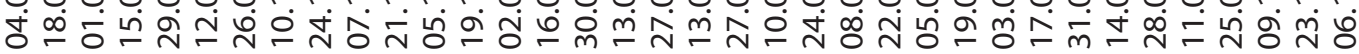

Date

- Treatment decision within 2 hours — Average — UCL and LCL

Fig 3. Scarborough emergency department assessment and decision. a) Proportion of patients commencing first assessment within 30 minutes of arrival to emergency department. b) Proportion of patients marked with treatment decision within 2 hours. $L C L=$ lower control limit; $U C L=$ upper control limit. 
Fig 4. Model predicated on co-location wherever possible of all acute services. $\mathrm{HCP}=$ healthcare provider; ITU = intensive treatment unit;

UTC $=$ urgent treatment centre.

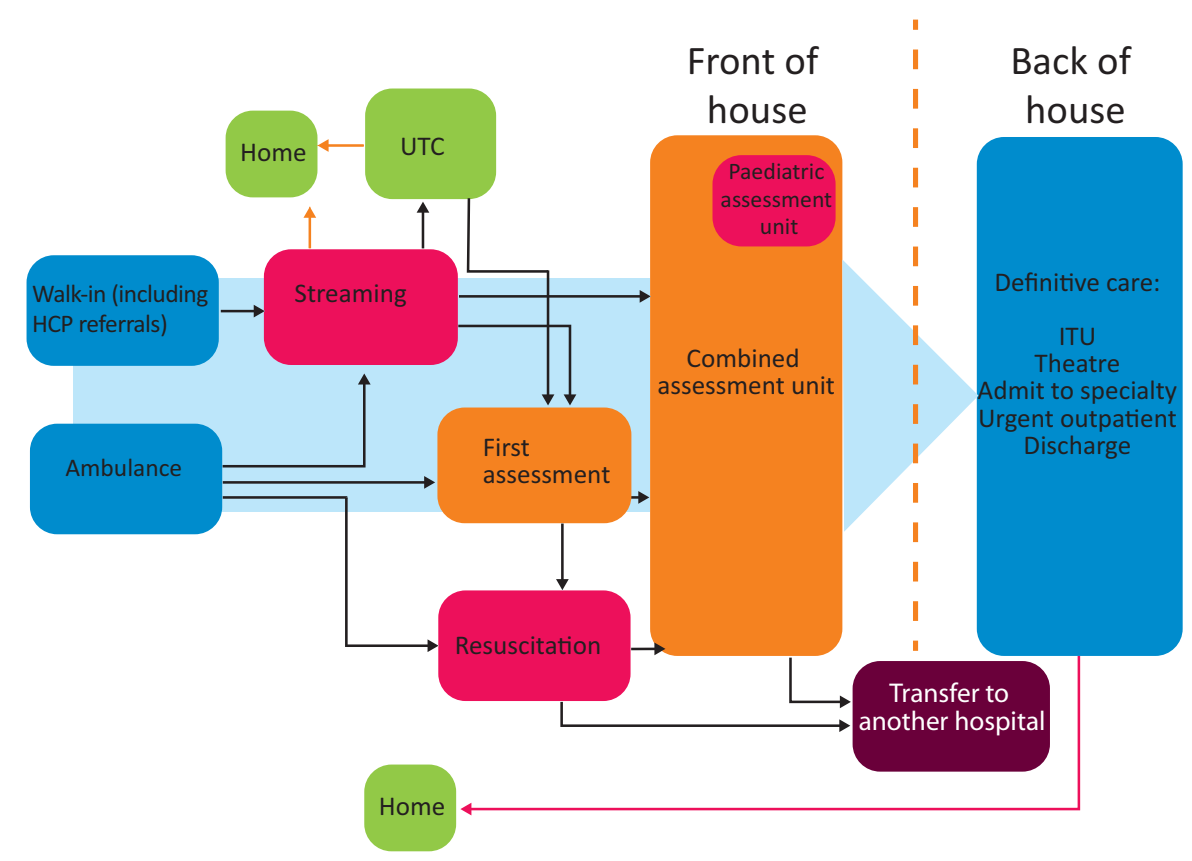

> Smaller systems have a more limited overall resource in terms of expertise to assess and treat the undifferentiated unwell patient. It makes sense to concentrate that expertise in a smaller physical space to increase the likelihood of having appropriate skills present in the clinical environment at any one time.

$>$ A single location of the unwell patient is effective in managing the increasing complexity of patients presenting over time. An example of this is the frail elderly patient who has experienced trauma, but who may have had a medical reason for their collapse or fall. In a system where such patients are sent to geographically distinct areas there is reduced opportunity for different specialists to easily manage the same patient at the same time (in this case, emergency medicine, orthopaedics and frailty specialists may all be needed to achieve an optimum outcome). The practitioner goes to the patient rather than the other way around and care is delivered in parallel rather than in series.

At present our estate is of a traditional configuration with a separate ED, acute medical unit and SDEC unit. There is a plan for a new-build which is in process at present and we would look to co-locate services in order to further support our model of care at that point. There is a 'chicken and egg' aspect to this, in that we need colleagues to work differently in order to deliver care in a new unit but the presence of a different geography will significantly assist in making that happen (Fig 4).

\section{Summary}

Smaller hospitals and systems have a workforce with greater limitations on their medical expertise and resources. Many are located in remote, rural or coastal locations, distant from specialist centres, often with a deprived and/or elderly population with a greater need for acute care. As a result, traditional models of medical care with specialists working independently of each other are difficult to maintain. There is a danger that without closer working between specialty colleagues it will be difficult to maintain standards of quality and safety, and deliver the standard of care that our patients deserve.

The potential solutions to these challenges that have been developed in our system are pragmatic, potential ways forward to deliver an appropriate quality of acute care in a challenged environment. They have been implemented using quality improvement methodology and we are continually evaluating and adapting in response to evidence that we are obtaining about the benefits of working in this way. We are also constantly learning from colleagues elsewhere who have implemented similar responses to similar difficulties in their own systems and we believe it is only by this form of collaborative working that we can strive to improve in an increasingly challenging healthcare environment.

\section{References}

1 Monitor. Facing the future: smaller acute providers. Monitor, 2014. https://assets.publishing.service.gov.uk/government/ uploads/system/uploads/attachment_data/file/320075/ smalleracuteproviders-report.pdf

2 Care Quality Commission. The state of care in NHS acute hospitals: 2014 to 2016. CQC, 2017. www.cqc.org.uk/sites/default/ files/20170302b_stateofhospitals_web.pdf [Accessed 17 October 2019].

3 Palmer B, Appleby J, Spencer J. Rural health care: A rapid review of the impact of rurality on the costs of delivering health care. London: Nuffield Health, 2019 [Accessed 17 October 2019].

4 Vaughan L, Edwards N, Imison C, Collins B. Rethinking acute medical care in smaller hospitals. London: Nuffield Trust, 2018 [Accessed 17 October 2019].

5 Emergency Care Intensive Support Team. Rapid assessment and treatment models in emergency departments. NHS, 2012. www. england.nhs.uk/wp-content/uploads/2013/08/rap-assess-treat.pdf [Accessed 05 December 2019].

6 The Royal College of Emergency Medicine. Initial assessment of emergency department patients. London: RCEM, 2017. www.rcem. ac.uk/docs/SDDC\% 20Intial \% 20Assessment \% 20(Feb \% 202017). pdf [Accessed 17th October 2019].

Address for correspondence: Dr Ed Smith, Scarborough Hospital, Woodlands Drive, Scarborough, North Yorkshire Y012 6QL, UK.

Email:edsmith@doctors.org.uk 